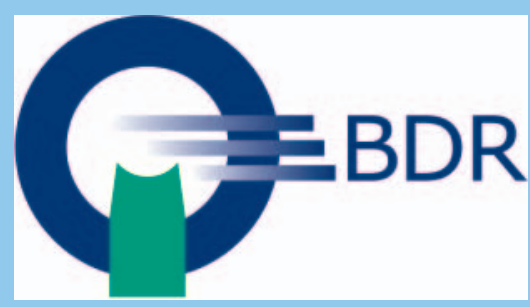

\title{
„Fortbildung 2.0 - wie Corona auch unseren beruflichen Alltag verändert“
}

Die notwendige Absage weitgehend aller Kongresse und Fortbildungsveranstaltungen seit dem Frühjahr dieses Jahres hat zunächst vor allem viele Fragen aufgeworfen, wie es denn weitergehen könnte. Inzwischen sind die meisten dieser ehemaligen Groß- und Riesenevents im digitalen Alltag angekommen. In besonders beeindruckender Form ist dies dem Deutschen Röntgenkongress in seiner Version „RöKo Digital 2020“ gelungen. Trotz der nur kurzen Vorbereitungszeit konnte aufgrund jahrelanger Vorarbeiten ein attraktives und umfangreiches Programm zusammengestellt werden.

Mittlerweile kommen auch die Vorzüge eines digitalen Konzeptes mehr und mehr zum Vorschein. Durch die zeitliche Streckung über viele Monate entfallen Parallelveranstaltungen und die Not, sich für eine der Alternativen zu entscheiden. So erreichen beim Präsenzkongress in der Regel Veranstaltungen in digitalen Zeiten um die 300 Teilnehmer. Während an Großkongressen sonst nur etwa die Hälfte der Mitarbeiterinnen und Mitarbeiter einer Institution teilnehmen kann, steht die Veranstaltung jetzt allen offen. Die Möglichkeit, Fragen schriftlich zu stellen und sich nicht öffentlich gleichsam outen zu müssen, beflügelt - wie bereits bei internationalen Veranstaltungen wie IROS, ECR und RSNA erprobt - die Fragefreudigkeit. Und auch auf den Kongressen eher als Randgebiete gesetzte Themen wie die Berufspolitik werden nach Umfragen durchaus nachgefragt und vermisst.

Die digitale Fortbildungsform strahlt jedoch weiter aus - auch über Großkongresse hinaus. So war es vor Jahren noch unvorstellbar, dass etwa die Aktualisierung der Fachkunde im Strahlenschutz digital erworben werden könnte. In Zeiten wie diesen ist es vollständig online möglich. Und auch Mitgliederversammlungen von etwa Arbeitsgemeinschaften der DRG und Landesverbänden des BDR erfreuen sich deutlich höheren Zuspruchs als in rein analogen Zeiten.

Fehlt also nichts? Doch, man muss sein Zeitmanagement dem digitalen Angebot anpassen. Ist man früher gereist, muss man heute zumindest die Zeit der Veranstaltung freihalten, sonst geht der digitale Kongress im Alltag an einem vorbei. Und es fehlt der persönliche Kontakt für Referenten und Teilnehmer! Für die echte interaktive Diskussion Auge in Auge, wie sie jede wissenschaftliche Präsentation befruchtet. Für die jungen Kolleginnen und Kollegen, die sich ins Gespräch bringen und für künftige Führungsaufgaben empfehlen wollen. Für das Gespräch mit den Kollegen, mit denen man zum Teil jahrelang gearbeitet hat und die man jetzt nur noch 1-mal im Jahr anlässlich einer Fortbildungsveranstaltung trifft. Für das Gespräch zwischen Kunde und Industrievertreter, in dem auch die leisen Zwischentöne für beide Seiten von enormem Gewicht sind. Für das berufspolitische 4-Augen-Gespräch, um Kompromisse zwischen unterschiedlichen Interessengruppen diskret auszuloten. Und natürlich sind nicht alle Berufs- und Altersgruppen trotz Tätigkeit in einem technischen Fach gleichermaßen affin für die digitalen Angebote.

So bleibt als Fazit festzustellen, dass die notgedrungen erfolgte Transformation der Kongresse und Weiterbildungsveranstaltungen für die reine Übermittlung medizinisch-sachlicher Fortbildung weitgehend gelungen ist. Die verbliebenen Wermutstropfen für wissenschaftliche Präsentationen, interaktive Diskussionen und berufspolitische Sondierungen müssen in den kommenden Monaten und Jahren konstruktiven Lösungen zugeführt werden. Konzepte hierzu gibt es inzwischen viele. Allen ist gemein, dass unser Kongress- und Fortbildungsalltag künftig ein anderer sein wird als bis zum März 2020. Der BDR wird den Prozess weiterhin konstruktiv begleiten und zusammen mit den übrigen beteiligten Partnern positiv gestalten.

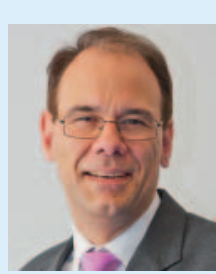

Prof. Dr. med. Hermann Helmberger, München 